\title{
Zero Capacity Region of Multidimensional Run Length Constraints
}

\author{
Hisashi Ito \\ Department of Information Science \\ Toho University, Chiba 274-8510, Japan \\ his@kuro.is.sci.toho-u.ac.jp \\ Akiko Kato \\ Dept. of Mathematical Engineering and Information Physics \\ University of Tokyo, Tokyo 113-8656, Japan \\ akiko@misojiro.t.u-tokyo.ac.jp \\ Zsigmond Nagy \\ Department of Electrical and Computer Engineering \\ University of California, San Diego, CA 92093-0407 \\ nagy@code.ucsd.edu \\ Kenneth Zeger \\ Department of Electrical and Computer Engineering \\ University of California, San Diego, CA 92093-0407 \\ zeger@ucsd.edu
}

Submitted: April 24, 1999; Accepted: September 1, 1999. 1991 Mathematics Subject Classification: 94A99, 58F03.

\footnotetext{
${ }^{0}$ This work was supported in part by the National Science Foundation and by a JSPS Fellowship for Young Scientists. A portion of this work was presented in Japanese at the Research Institute for Mathematical Sciences Workshop (RIMS Kokyuroku), Kyoto University, Japan, January 1999.
} 


\begin{abstract}
For integers $d$ and $k$ satisfying $0 \leq d \leq k$, a binary sequence is said to satisfy a one-dimensional $(d, k)$ run length constraint if there are never more than $k$ zeros in a row, and if between any two ones there are at least $d$ zeros. For $n \geq 1$, the $n$-dimensional $(d, k)$-constrained capacity is defined as

$$
C_{d, k}^{(n)}=\lim _{m_{1}, m_{2}, \ldots, m_{n} \rightarrow \infty} \frac{\log _{2} N_{m_{1}, m_{2}, \ldots, m_{n}}^{(n ; d, k)}}{m_{1} m_{2} \cdots m_{n}}
$$

where $N_{m_{1}, m_{2}, \ldots, m_{n}}^{(n ; d, k)}$ denotes the number of $m_{1} \times m_{2} \times \cdots \times m_{n} n$-dimensional binary rectangular patterns that satisfy the one-dimensional $(d, k)$ run length constraint in the direction of every coordinate axis. It is proven for all $n \geq 2$, $d \geq 1$, and $k>d$ that $C_{d, k}^{(n)}=0$ if and only if $k=d+1$. Also, it is proven for every $d \geq 0$ and $k \geq d$ that $\lim _{n \rightarrow \infty} C_{d, k}^{(n)}=0$ if and only if $k \leq 2 d$.
\end{abstract}




\section{Introduction}

A binary sequence is $(d, k)$-constrained (or "runlength constrained") if there are at most $k$ consecutive zeros and between every two ones there are at least $d$ consecutive zeros. An $n$-dimensional pattern of zeros and ones arranged in an $m_{1} \times m_{2} \times \cdots \times m_{n}$ hyper-rectangle is $(d, k)$-constrained if it is (1-dimensional) $(d, k)$-constrained in each of the $n$ coordinate axis directions. The $n$-dimensional $(d, k)$-capacity is defined as

$$
C_{d, k}^{(n)}=\lim _{m_{1}, m_{2}, \ldots, m_{n} \rightarrow \infty} \frac{\log _{2} N_{m_{1}, m_{2}, \ldots, m_{n}}^{(n ; d, k)}}{m_{1} m_{2} \cdots m_{n}},
$$

where $N_{m_{1}, m_{2}, \ldots, m_{n}}^{(n ; d, k)}$ denotes the number of $(d, k)$-constrained patterns on an $m_{1} \times m_{2} \times$ $\cdots \times m_{n}$ hyper-rectangle. A simple proof was given in [5] that shows the existence of two-dimensional $(d, k)$-capacities, and a slight modification of the proof can show that the $n$-dimensional $(d, k)$-capacities exist. The capacity $C_{d, k}^{(n)}$ represents the maximum number of bits of information that can be stored asymptotically per unit volume in $n$-dimensional space without violating the $(d, k)$ constraint.

The study of 1 -dimensional $(d, k)$-capacities was originally motivated by applications in magnetic storage. Interest in 2-dimensional $(d, k)$-capacities has recently increased due to emerging 2-dimensional optical recording devices, and the 3-dimensional $(d, k)$-capacities may play a role in future applications as well. A tutorial on these topics is given in [4]. Capacities in four and higher dimensions yield natural generalizations of interesting mathematical questions in lower dimensions.

In general, the exact values of the various $n$-dimensional $(d, k)$-capacities are not known except in a few cases [6]. For example, in all dimensions, if $k=d$ the capacity is zero, and if $d=0$ the capacity is positive for all $k \geq 1$. In one dimension the capacity is positive whenever $k>d \geq 0$. The capacity is known to be a monotonically nonincreasing function of $n$ and $d$ and a monotonically nondecreasing function of $k$. It was recently shown [5] that whenever $k>d \geq 1$, the 2-dimensional capacity is zero if and only if $k=d+1$. These facts are summarized in our Lemma 1 .

Some interesting facts are known about the capacities for $d=0$ and $k=1$ in three and lower dimensions. In one dimension, $N_{m}^{(1 ; 0,1)}$ is known [6] to be a Fibonacci sequence with initial conditions $N_{1}^{(1 ; 0,1)}=2$ and $N_{2}^{(1 ; 0,1)}=3$, and thus the 1-dimensional $(0,1)$-capacity is the logarithm of the golden mean, namely $C_{0,1}^{(1)}=\log _{2} \frac{1+\sqrt{5}}{2} \approx 0.694$. Very tight upper and lower bounds on the $(0,1)$-capacity were given for two dimensions in [2] and for three dimensions in [7]. These two and three dimensional $(0,1)$-capacities are $C_{0,1}^{(2)} \approx 0.58789116$ and $C_{0,1}^{(3)} \approx 0.52$, given here to their known accuracies.

In this paper we present two main results that characterize the zero capacity region for finite dimensions and in the limit of large dimensions. The first result generalizes the zero capacity characterization in [5] to all dimensions greater than one. Namely it gives a necessary and sufficient condition on $d$ and $k$ for the capacity to equal zero. This condition turns out to be exactly the same as in dimension 2 . The second 
result gives a necessary and sufficient condition on $d$ and $k$, such that the capacity approaches zero in the limit as the dimension $n$ grows to infinity. These results are summarized in the following two theorems.

Theorem 1 For every $n \geq 2, d \geq 1$, and $k>d$,

$$
C_{d, k}^{(n)}=0 \Leftrightarrow k=d+1 .
$$

Theorem 2 For every $d \geq 0$ and $k \geq d$,

$$
\lim _{n \rightarrow \infty} C_{d, k}^{(n)}=0 \Leftrightarrow k \leq 2 d
$$

The following lemma contains useful facts about capacities for various constraints and is used to establish Theorems 1 and 2 .

\section{Lemma 1}

(a) $C_{d, k+1}^{(n)} \geq C_{d, k}^{(n)} ; \quad$ whenever $n \geq 1,0 \leq d \leq k$

(b) $C_{d, k}^{(n)} \geq C_{d+1, k}^{(n)} ; \quad$ whenever $n \geq 1,0 \leq d<k$

(c) $C_{d, k}^{(n+1)} \leq C_{d, k}^{(n)} ; \quad$ whenever $n \geq 1,0 \leq d<k$

(d) $C_{d, d}^{(n)}=0 ; \quad$ whenever $n \geq 1, d \geq 0$

(e) $C_{d, 2 d+1}^{(n)} \geq \frac{1}{2(d+1)} ; \quad$ whenever $n \geq 1, d \geq 0$

(f) $C_{0, k}^{(n)}>0$; whenever $n \geq 1, k \geq 1$

(g) $C_{d, k}^{(1)}>0$; whenever $0 \leq d<k$

(h) $C_{d, k}^{(2)}=0$ if and only if $k=d+1$; whenever $1 \leq d<k$.

Proof.

(a) Follows from the fact that $N_{m_{1}, m_{2}, \ldots, m_{n}}^{(n ; d, k+1)} \geq N_{m_{1}, m_{2}, \ldots, m_{n}}^{(n ; d, k)}$ since any pattern that satisfies the $(d, k)$ constraint also satisfies the $(d, k+1)$ constraint.

(b) Follows from $N_{m_{1}, m_{2}, \ldots, m_{n}}^{(n ; d, k)} \geq N_{m_{1}, m_{2}, \ldots, m_{n}}^{(n ; d+1, k)}$.

(c)

$$
\begin{aligned}
C_{d, k}^{(n+1)} & =\lim _{m_{1}, m_{2}, \ldots, m_{n+1} \rightarrow \infty} \frac{\log _{2} N_{m_{1}, m_{2}, \ldots, m_{n+1}}^{(n+1 ; d, k)}}{m_{1} m_{2} \ldots m_{n+1}} \\
& \leq \lim _{m_{1}, m_{2}, \ldots, m_{n+1} \rightarrow \infty} \frac{\log _{2}\left(N_{m_{1}, m_{2}, \ldots, m_{n}}^{(n ; d, k)}\right)^{m_{n+1}}}{m_{1} m_{2} \ldots m_{n+1}}=\lim _{m_{1}, m_{2}, \ldots, m_{n} \rightarrow \infty} \frac{\log _{2} N_{m_{1}, m_{2}, \ldots, m_{n}}^{(n ; d, k)}}{m_{1} m_{2} \ldots m_{n}} \\
& =C_{d, k}^{(n)} .
\end{aligned}
$$


(d) $C_{d, d}^{(1)}=0$ since $N_{m}^{(1 ; d, d)} \leq d+1$. The result then follows by induction and from the monotonicity in part (c).

(e) Let $T=\{1,2, \ldots, m\}$, where $m$ is a multiple of $2(d+1)$. Any mapping $f: T^{n} \rightarrow\{0,1\}$ satisfying $f\left(x_{1}, x_{2}, \ldots, x_{n}\right)=1$ when $2(d+1)$ divides $\sum_{i=1}^{n} x_{i}$, and $f\left(x_{1}, x_{2}, \ldots, x_{n}\right)=0$ when $d+1$ does not divide $\sum_{i=1}^{n} x_{i}$, induces a $(d, 2 d+$ $1)$-constrained pattern on $T^{n}$. Since the value of $f\left(x_{1}, x_{2}, \ldots, x_{n}\right)$ can be chosen arbitrarily when $\sum_{i=1}^{n} x_{i} \equiv(d+1) \bmod 2(d+1)$, the number of $(d, 2 d+1)$-constrained patterns on $T^{n}$ is at least $2^{m^{n} /(2(d+1))}$ and hence $N_{m, m, \ldots, m}^{(n ; d, 2 d+1)} \geq 2^{m^{n} /(2(d+1))}$. Thus

$$
C_{d, 2 d+1}^{(n)} \geq \lim _{m \rightarrow \infty} \frac{m^{n} /(2(d+1))}{m^{n}}=\frac{1}{2(d+1)} .
$$

(f) Follows from (a) and (e).

(g) It is known [1] that $C_{d, \infty}^{(1)}=C_{d-1,2 d-1}^{(1)}$ for $d \geq 1$, and also that for $0 \leq d<k<$ $\infty$, the 1-dimensional capacity is the logarithm (base 2 ) of the largest real root of the equation $X^{k+1}-X^{k-d}-X^{k-d-1}-\cdots-X-1=0$. The equation clearly has a root greater than 1 , and thus the result follows.

(h) This was proven in [5].

\section{Proof of Theorem 1}

Proof. Lemma 1(c),(h) shows that $C_{d, d+1}^{(n)}=0$ for all $d \geq 1$ and all $n \geq 2$. To prove $C_{d, k}^{(n)}>0$ for $k \geq d+2$, it suffices by Lemma $1(\mathrm{a}),(\mathrm{h})$ to prove $C_{d, d+2}^{(n)}>0$ for all $d \geq 1$ and $n \geq 3$. This is shown below in Proposition 1 for even $d \geq 0$, and in Proposition 2 for odd $d \geq 3$. A special case of Lemma 1(e) shows the result for $d=1$ and for all $n \geq 3$. This completes the proof of Theorem 1 .

The following definitions are useful for proving Propositions 1 and 2 . Let $S=\{0,1, \ldots$, $d+1\}$. The set $S^{n}$ is an $n$-cube, and any mapping $g: S^{n} \rightarrow\{0,1\}$ is a binary $n$-cube. A row of an $n$-cube is any set of the form $\left\{\left(c_{1}, \ldots, c_{l-1}, x, c_{l+1}, \ldots, c_{n}\right): x \in S\right\}$ for some fixed $l$, and some fixed $c_{j} \in S$ for $j=1, \ldots, l-1, l+1, \ldots, n$. A binary $n$-cube $g$ is a permutation $n$-cube if $g$ equals 1 once per row of $S^{n}$.

A binary $n$-cube $g$ is $(d, d+2)$-constrained unless $g$ takes the value one twice on some consecutive $d$ points in some row of $S^{n}$. It is clear that permutation $n$-cubes are $(d, d+2)$-constrained. A set of permutation $n$-cubes is $(d, d+2)$-compatible if the concatenation of any two of the cubes along a face (i.e. with translation but without rotation) is also $(d, d+2)$-constrained. If $S_{1}, \ldots, S_{n}$ are subsets of $S$, each consisting of two consecutive integers, the smaller of which is even, then $S_{1} \times \cdots \times S_{n}$ 
is a $b i$-subcube of $S^{n}$. If a permutation $n$-cube $g$ equals 1 exactly once per row in a bi-subcube, then the restriction of $g$ to the bi-subcube is said to be a permutation bi-subcube.

A binary $n$-cube $h$ is a reversal of a permutation $n$-cube $g$ if $h$ equals $1-g$ on the members of a (possibly empty) subset of all the bi-subcubes in $S^{n}$, on each of which $g$ is a permutation bi-subcube, and $h$ equals $g$ elsewhere. A reversal $h$ of any permutation cube $g$ is also a permutation cube, and $g$ and $h$ together form a $(d, d+2)$-compatible set. More generally, any collection of reversals of a given permutation $n$-cube forms a $(d, d+2)$-compatible set (see Lemma 2). In Propositions 1 and 2, we construct a $(d, d+2)$-compatible family of reversals of a certain permutation $n$-cube, and then obtain a lower bound on the $(d, d+2)$-capacity from the cardinality of the family.

A mapping $\bar{f}: S^{n} \rightarrow S$ is a latin $n$-cube if on every row of $S^{n}, \bar{f}$ is a permutation of $S$. This definition is a generalization of a latin square, although alternate definitions have been given in [3]. For any permutation $n$-cube $g$, any $l<n$, and any $c_{j} \in S$ (for $j=1, \ldots, l-1, l+1, \ldots, n-1$ ), the relation $x \mapsto y$ determined by $g\left(c_{1}, \ldots, c_{l-1}, x, c_{l+1}, \ldots, c_{n-1}, y\right)=1$ is a permutation. This leads us to define a correspondence between permutation $n$-cubes and latin $(n-1)$-cubes as follows. Let $g: S^{n} \rightarrow\{0,1\}$ be a permutation $n$-cube and for each $\left(x_{1}, x_{2}, \ldots, x_{n-1}\right) \in S^{n-1}$, let $y\left(x_{1}, \ldots, x_{n-1}\right)$ be the unique element of $S$ such that $g\left(x_{1}, x_{2}, \ldots, x_{n-1}, y\left(x_{1}, \ldots, x_{n-1}\right)\right)=$ 1. Then the mapping $\bar{g}: S^{n-1} \rightarrow S$ defined by $\bar{g}\left(x_{1}, x_{2}, \ldots, x_{n-1}\right)=y\left(x_{1}, \ldots, x_{n-1}\right)$ is a latin $(n-1)$-cube, and the correspondence $g \mapsto \bar{g}$ is bijective (see Lemma 2). The bar notation will be exclusively used for latin cubes. For any integers $a \geq 0$ and $b>0$, we use the notation " $a \bmod b$ " to mean the unique integer $a-\left\lfloor\frac{a}{b}\right\rfloor b$.

Lemma 2 Let $\bar{e}_{n}: S^{n} \rightarrow S$ be a sequence of mappings defined recursively for $n \geq 3$ by

$$
\bar{e}_{n}\left(x_{1}, \ldots, x_{n}\right)=\bar{e}_{2}\left(\bar{e}_{n-1}\left(x_{1}, \ldots, x_{n-1}\right), x_{n}\right)
$$

where $\bar{e}_{2}$ is a latin square. Then $\bar{e}_{n}$ is a latin $n$-cube for all $n \geq 2$, and the set of all reversals of the corresponding permutation $(n+1)$-cube $e_{n}$ is $(d, d+2)$-compatible.

Proof. Use induction on $n$. Assume $\bar{e}_{2}, \ldots, \bar{e}_{n-1}$ are latin cubes (for $n \geq 3$ ) and fix all but one of the arguments $x_{1}, \ldots, x_{n}$ of $\bar{e}_{n}$. If $x_{1}, \ldots, x_{n-1}$ are fixed then $\bar{e}_{n}$ is a permutation of $S$ since fixing the first argument of $\bar{e}_{2}$ yields a permutation of $S$. Likewise, if $x_{n}$ and all but one of $x_{1}, \ldots, x_{n-1}$ are fixed, then by the induction hypothesis $\bar{e}_{n-1}\left(x_{1}, \ldots, x_{n-1}\right)$ is a permutation of $S$ and $\bar{e}_{2}$ is a permutation of $S$ since its second argument $x_{n}$ is fixed. Thus $\bar{e}_{n}$ is a latin $n$-cube.

Let $h$ be a binary $(n+1)$-cube $h: S^{n+1} \rightarrow\{0,1\}$ satisfying

$$
h\left(x_{1}, \ldots, x_{n+1}\right)= \begin{cases}1 & \text { if } x_{n+1}=\bar{e}_{n}\left(x_{1}, \ldots, x_{n}\right) \\ 0 & \text { otherwise }\end{cases}
$$


Then $h$ is a permutation $(n+1)$-cube since $\bar{e}_{n}$ is a latin $n$-cube, and $\bar{h}=\bar{e}_{n}$ from the definition of the bar notation. This shows that there exists a unique permutation $(n+1)$-cube $h$ (i.e. $\left.e_{n}\right)$ corresponding to the latin $n$-cube $\bar{e}_{n}$.

The permutation $(n+1)$-cube $e_{n}$ has rows of length $d+2$, each containing a single one. For any collection of bi-subcubes, on each of which $e_{n}$ is a permutation bi-subcube, any row of $S^{n+1}$ can intersect at most one of these bi-subcubes. This implies that any facewise concatenation of any two reversals of $e_{n}$ will only have pairs of ones at distances $d, d+1$, or $d+2$ apart, and thus any set of reversals of $e_{n}$ is $(d, d+2)$-compatible.

Proposition 1 For every $n \geq 2$ and every even $d \geq 0$,

$$
C_{d, d+2}^{(n)} \geq \frac{1}{2^{n-1}(d+2)}
$$

Proof. Define a mapping $\bar{e}_{2}: S^{2} \rightarrow S$ such that

$$
\bar{e}_{2}\left(x_{1}, x_{2}\right)=\left\{\begin{array}{cl}
\left(x_{1}+x_{2}-2\right) \bmod (d+2) & \text { if } x_{1} \text { and } x_{2} \text { are odd } \\
\left(x_{1}+x_{2}\right) \bmod (d+2) & \text { otherwise }
\end{array}\right.
$$

as in Figure 1. The mapping $\bar{e}_{2}$ is a latin square since $\bar{e}_{2}$ is a permutation of the set $S$ when either the first or second component is held fixed. For each $n \geq 3$, use (1) to recursively define the latin $n$-cube $\bar{e}_{n}: S^{n} \rightarrow S$.

For each $n \geq 2$, let $x_{1}, \ldots, x_{n}$ be any set of even integers from $S$. We claim that for any $y_{1}, \ldots, y_{n} \in\{0,1\}$,

$$
\bar{e}_{n}\left(x_{1}+y_{1}, \ldots, x_{n}+y_{n}\right)=\left\{\begin{aligned}
\left(x_{1}+\cdots+x_{n}\right) \bmod (d+2) & \text { if } \sum_{i=1}^{n} y_{i} \text { is even } \\
\left(1+x_{1}+\cdots+x_{n}\right) \bmod (d+2) & \text { if } \sum_{i=1}^{n} y_{i} \text { is odd }
\end{aligned}\right.
$$

To prove this claim, use induction on $n$. It is easy to see from (2) that the claim is true for $n=2$. By (1) and the induction hypothesis,

$$
\begin{aligned}
& \bar{e}_{n}\left(x_{1}+y_{1}, \ldots, x_{n}+y_{n}\right)= \\
& \left\{\begin{aligned}
\bar{e}_{2}\left(\left(x_{1}+\cdots+x_{n-1}\right) \bmod (d+2), x_{n}+y_{n}\right) & \text { if } \sum_{i=1}^{n-1} y_{i} \text { is even } \\
\bar{e}_{2}\left(\left(1+x_{1}+\cdots+x_{n-1}\right) \bmod (d+2), x_{n}+y_{n}\right) & \text { if } \sum_{i=1}^{n-1} y_{i} \text { is odd }
\end{aligned}\right.
\end{aligned}
$$

Equivalently, when $\sum_{i=1}^{n} y_{i}$ is even

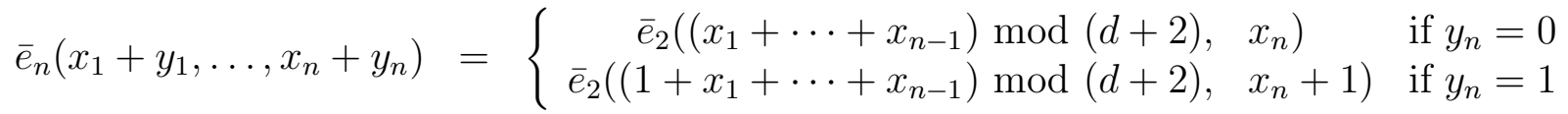

$$
\begin{aligned}
& =\left(x_{1}+\cdots+x_{n}\right) \bmod (d+2) \text {, }
\end{aligned}
$$


and when $\sum_{i=1}^{n} y_{i}$ is odd

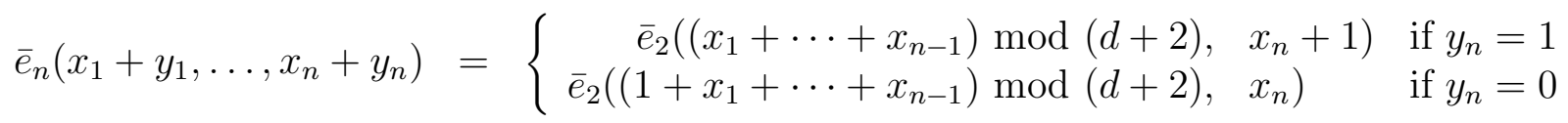

$$
\begin{aligned}
& =\left(1+x_{1}+\cdots+x_{n}\right) \bmod (d+2) \text {, }
\end{aligned}
$$

thus proving the claim.

The claim just proved implies that the corresponding permutation $(n+1)$-cube $e_{n}$ satisfies

$$
e_{n}\left(x_{1}+y_{1}, \ldots, x_{n+1}+y_{n+1}\right)= \begin{cases}1 & \text { if } \sum_{i=1}^{n+1} y_{i} \text { is even } \\ 0 & \text { if } \sum_{i=1}^{n+1} y_{i} \text { is odd }\end{cases}
$$

for any even integers $x_{1}, \ldots, x_{n+1} \in S$ such that $x_{n+1}=\sum_{i=1}^{n} x_{i} \bmod (d+2)$, and for any $y_{1}, \ldots, y_{n+1} \in\{0,1\}$. Thus the restriction of $e_{n}$ to each bi-subcube $\left\{\left(x_{1}+\right.\right.$ $\left.\left.y_{1}, \ldots, x_{n+1}+y_{n+1}\right): y_{1}, \ldots, y_{n+1} \in\{0,1\}\right\}$ is a permutation bi-subcube. Then the cardinality of the set of all reversals of $e_{n}$ is $2^{\left(\frac{d+2}{2}\right)^{n}}$, and Lemma 2 gives the lower bound

$$
C_{d, d+2}^{(n)} \geq \frac{\log _{2} 2^{\left(\frac{d+2}{2}\right)^{n-1}}}{(d+2)^{n}}=\frac{1}{2^{n-1}(d+2)}
$$

Proposition 2 For every $n \geq 2$ and every odd $d \geq 3$,

$$
C_{d, d+2}^{(n)} \geq \frac{1}{(d+2)^{n}}\left(\begin{array}{c}
n-1+\frac{d-3}{2} \\
n-1
\end{array}\right)
$$

Proof.

Define a mapping $\bar{e}_{2}: S^{2} \rightarrow S$ such that

$$
\bar{e}_{2}\left(x_{1}, x_{2}\right)=\left\{\begin{aligned}
x_{1}+x_{2}-2 & \text { if } x_{1} \text { and } x_{2} \text { are odd } \\
x_{1}+x_{2} & \text { otherwise }
\end{aligned}\right.
$$

for $2\left\lfloor\frac{x_{1}}{2}\right\rfloor+2\left\lfloor\frac{x_{2}}{2}\right\rfloor \leq d-3$. The values of $\bar{e}_{2}$ for $2\left\lfloor\frac{x_{1}}{2}\right\rfloor+2\left\lfloor\frac{x_{2}}{2}\right\rfloor>d-3$ (i.e. below the bold 2-step staircase line in Figures 2 and 3) are defined as follows. The points on the diagonal line above the main diagonal have value $d$, as does the bottom right corner of the square. Thus, $d$ appears once in each row and in each column in the square. The portion of the next higher diagonal that lies below the 2-step staircase line has value $d-1$. The area below and including the main diagonal of the square, except the bottom row and the rightmost column, is partitioned into diagonal strips of width 4 . Each diagonal strip is formed by repeating the staircase pattern shape of

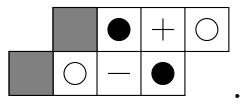


The bottom row is formed by repeating the pattern

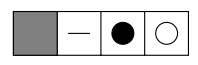

and the rightmost column is formed by repeating the pattern

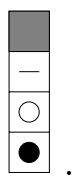

(For the case $d \equiv 1 \bmod 4$ the bottom-rightmost diagonal strip is truncated at width 3, and the above patterns are cut off accordingly, as illustrated in Figure 2.) Within any given diagonal strip, all labels containing a particular symbol represent the same integer. In particular, in the $j$ th diagonal, (for $j=1,2, \ldots,\left\lfloor\frac{d}{4}\right\rfloor+1$ ), the square labels $\square, \square, \square, \square$, and $\square$ represent $4 j-2,4 j-3,4 j-4,4 j-5$, and $4 j-6$ respectively (for $j=1, \square$ and $\square$ represent $d-1$ and $d+1$, respectively). For any $i \in\{0,1, \ldots, d-2\}$ it can be seen that the value $i$ appears once in every row and column of the top left $2\left\lfloor\frac{i}{2}\right\rfloor+2$ rows and columns, and the value $i$ appears once in every row and column of the bottom right $d-2\left\lfloor\frac{i}{2}\right\rfloor$ rows and columns. Also, the main diagonal of $S^{2}$ contains only the value $d+1$, and the value $d-1$ appears in the rightmost column at $\left(x_{1}, x_{2}\right)=(1, d+2)$, in the bottom row at $\left(x_{1}, x_{2}\right)=(d+2,1)$, and in alternating positions on the diagonals that lie two above and two below the main diagonal of $S^{2}$. The value $d-1$ appears in the rightmost column at $\left(x_{1}, x_{2}\right)=(1, d+2)$ and in the bottom row at $\left(x_{1}, x_{2}\right)=(d+2,1)$, and these points do not lie on the diagonals two below nor two above the main diagonal. Consequently, every number $0,1, \ldots, d+1$ appears exactly once in each row and in each column in the original $(d+2) \times(d+2)$ square $S^{2}$, showing that $\bar{e}_{2}$ is a latin square.

Using (1) and the definition of $\bar{e}_{2}$ just given, recursively define for each integer $n \geq 3$, the latin $n$-cube $\bar{e}_{n}: S^{n} \rightarrow S$. For any $n \geq 2$, if $x_{1}, \ldots, x_{n}$ are even integers from $S$ such that $\sum_{i=1}^{n} x_{i} \leq d-3$, then for any $y_{1}, \ldots, y_{n} \in\{0,1\}$,

$$
\bar{e}_{n}\left(x_{1}+y_{1}, \ldots, x_{n}+y_{n}\right)=\left\{\begin{aligned}
x_{1}+\cdots+x_{n} & \text { if } \sum_{i=1}^{n} y_{i} \text { is even } \\
1+x_{1}+\cdots+x_{n} & \text { if } \sum_{i=1}^{n} y_{i} \text { is odd }
\end{aligned}\right.
$$

from the same proof as in Proposition 1, but with the added constraint $\sum_{i=1}^{n} x_{i} \leq d-3$.

As in Proposition 1, the set of reversals of the permutation $(n+1)$-cube $e_{n}$ is $(d, d+2)$-compatible. There are $\left(\begin{array}{c}n+\frac{d-3}{2} \\ n\end{array}\right)$ permutation bi-subcubes in this case and the volume of the $(n+1)$-cube $S^{n+1}$ (i.e. the domain of $\left.e_{n}\right)$ is $(d+2)^{n+1}$. Hence

$$
C_{d, d+2}^{(n)} \geq \frac{1}{(d+2)^{n}}\left(\begin{array}{c}
n-1+\frac{d-3}{2} \\
n-1
\end{array}\right)
$$




\section{Proof of Theorem 2}

Proof. Lemma 1(e) gives $\lim _{n \rightarrow \infty} C_{d, 2 d+1}^{(n)}>0$ for every $d \geq 0$, and thus $\lim _{n \rightarrow \infty} C_{d, k}^{(n)}>$ 0 for every $k \geq 2 d+1$ by Lemma 1(a). Lemma 3 below implies that $C_{d, k}^{(n)} \leq$ $\left(\frac{k-d}{k-d+1}\right)^{n-1} C_{d, k}^{(1)}$ whenever $d<k \leq 2 d$, and hence $\lim _{n \rightarrow \infty} C_{d, k}^{(n)}=0$. This together with Lemma 1(d) completes the proof of Theorem 2.

Lemma 3 If $n \geq 2$ and $1 \leq d<k \leq 2 d$ then

$$
C_{d, k}^{(n)} \leq \frac{k-d}{k-d+1} C_{d, k}^{(n-1)}
$$

Proof. Let $l$ and $m$ be positive integers and let $V=\{1,2, \ldots, m\}$. Define the following $n$-dimensional hyper-rectangles (for $j=1,2, \ldots, l$ ):

$$
\begin{aligned}
T & =\left\{\left(x_{1}, \ldots, x_{n-1}, x_{n}\right): x_{1}, \ldots, x_{n-1} \in V,-d \leq x_{n}<(k-d+1) l\right\} \\
U_{0} & =\left\{\left(x_{1}, \ldots, x_{n-1}, x_{n}\right): x_{1}, \ldots, x_{n-1} \in V,-d \leq x_{n}<0\right\} \\
U_{j} & =\left\{\left(x_{1}, \ldots, x_{n-1}, x_{n}\right): x_{1}, \ldots, x_{n-1} \in V,(k-d+1)(j-1)<x_{n}<(k-d+1) j\right\}
\end{aligned}
$$

and let $U=\bigcup_{j=0}^{l} U_{j}$. Note that there is a gap of width one between consecutive sets $U_{j}$ and $U_{j+1}$ (To help visualize the proof, the case of $n=3$ is illustrated in Figure 4). A binary mapping on $U$ is said to be $(d, k)$-constrained if it induces a $(d, k)$-constrained pattern on each $U_{j}$. Let $N_{T}$ and $N_{U_{j}}$ be the numbers of distinct $(d, k)$-constrained mappings on $T$ and $U_{j}$ (for $j=0,1, \ldots, l$ ), respectively. We show that $N_{T} \leq \prod_{j=0}^{l} N_{U_{j}}$.

To this end, it suffices to exhibit an injection from the set of all $(d, k)$-constrained mappings on $T$ to those on $U$. Thus we demonstrate that every $(d, k)$-constrained mapping on $T$ is completely determined by its restriction to $U$.

Assume the contrary. Then there exist two $(d, k)$-constrained mappings $f_{0}: T \rightarrow$ $\{0,1\}$ and $f_{1}: T \rightarrow\{0,1\}$ that agree on $U$ but differ on $T$. Let $\left(c_{1}, \ldots, c_{n-1}, c_{n}\right) \in T$ be such that $f_{0}\left(c_{1}, \ldots, c_{n-1}, c_{n}\right) \neq f_{1}\left(c_{1}, \ldots, c_{n-1}, c_{n}\right)$.

Since $f_{0}$ and $f_{1}$ agree on $U, c_{n}$ must be a multiple of $k-d+1$. Let $J$ be the smallest nonnegative integer $j$ such that $f_{0}\left(c_{1}, \ldots, c_{n-1},(k-d+1) j\right) \neq f_{1}\left(c_{1}, \ldots, c_{n-1},(k-\right.$ $d+1) j)$. Without loss of generality assume $f_{0}\left(c_{1}, \ldots, c_{n-1},(k-d+1) J\right)=0$ and $f_{1}\left(c_{1}, \ldots, c_{n-1},(k-d+1) J\right)=1$. Note that $(k-d+1)(J+1)-1 \leq(k-d+1) J+d$ since $k \leq 2 d$. Also, since $f_{1}\left(c_{1}, \ldots, c_{n-1},(k-d+1) J\right)=1, f_{1}$ must equal zero for at least $d$ consecutive positions next to this point. Thus $f_{1}\left(c_{1}, \ldots, c_{n-1}, x\right)=0$ for all $x$ in the range $(k-d+1) J-d \leq x<(k-d+1)(J+1)$, excluding $x=(k-d+1) J$. Therefore $f_{0}\left(c_{1}, \ldots, c_{n-1}, x\right)=0$ for this same set of $x$ 's, since either $\left(c_{1}, \ldots, c_{n-1}, x\right)$ is in $U$ or else because of the choice of $J$. But by assumption $f_{0}\left(c_{1}, \ldots, c_{n-1},(k-d+1) J\right)=0$, so a string of $k+1$ zeros in a row occurs for $f_{0}$ (from $x=(k-d+1) J-d$ to 
$x=(k-d+1)(J+1)-1)$ contradicting the $(d, k)$ constraint. This proves that every $(d, k)$-constrained mapping on $T$ is uniquely determined by its restriction to $U$. This establishes that $N_{T} \leq \prod_{j=0}^{l} N_{U_{j}}$.

Now, let $M$ denote the number of distinct $(d, k)$-constrained mappings on an $(n-1)$-dimensional hypercube of side length $m$. Clearly, $\prod_{j=0}^{l} N_{U_{j}} \leq M^{(k-d) l+d}$, since $N_{U_{0}} \leq M^{d}$ and $N_{U_{j}} \leq M^{k-d}$ for $j=1,2, \ldots, l$. Thus,

$$
\begin{aligned}
C_{d, k}^{(n)} & =\lim _{l, m \rightarrow \infty} \frac{\log _{2} N_{T}}{((k-d+1) l+d) m^{n-1}} \leq \lim _{l, m \rightarrow \infty} \frac{\log _{2} \prod_{j=0}^{l} N_{U_{j}}}{((k-d+1) l+d) m^{n-1}} \\
& \leq \lim _{l, m \rightarrow \infty} \frac{\log _{2} M^{(k-d) l+d}}{((k-d+1) l+d) m^{n-1}} \\
& =\lim _{l \rightarrow \infty} \frac{(k-d) l+d}{(k-d+1) l+d} \cdot \lim _{m \rightarrow \infty} \frac{\log _{2} M}{m^{n-1}}=\frac{k-d}{k-d+1} C_{d, k}^{(n-1)} .
\end{aligned}
$$

\section{Comments}

For $d=1$, Lemma $1(\mathrm{e})$ implies that $C_{1,3}^{(n)} \geq 1 / 4$ for $n \geq 3$. A more complicated proof can show that $C_{1,3}^{(n)} \geq C_{0,1}^{(n)} / 2$ for all $n \geq 2$ (note that $C_{0,1}^{(n)} \geq 1 / 2$ by Lemma $1(\mathrm{e})$ ). For odd $d \geq 3$ Proposition 2 gives $C_{d, d+2}^{(2)} \geq \frac{d-1}{2(d+2)^{2}}$ whereas a slightly better lower bound $C_{d, d+2}^{(2)} \geq \frac{d+1}{2(d+2)^{2}}$ was given in [5, Theorem 2]. Propositions 1 and 2 establish that $C_{d, d+2}^{(n)}>0$. Alternatively it is possible to prove $C_{d, d+2}^{(n)}>0$ in a simpler manner, but with weaker lower bounds on $C_{d, d+2}^{(n)}$ than those given in these propositions. 
THE ELECTRONiC JOURNAL of COMBinAtorics 6 (1999), \#R33

\section{References}

[1] J. J. Ashley and P. H. Siegel, "A Note on the Shannon Capacity of Run-LengthLimited Codes," IEEE Trans. Information Theory, vol. 33, no. 4, July 1987, pp. 601-605.

[2] N. J. Calkin and H. S. Wilf, "The Number of Independent Sets in a Grid Graph," SIAM J. on Discrete Mathematics, vol. 11, February 1998, pp. 54-60.

[3] J. Dénes and A. D. Keedwell, Latin Squares: New Developments in the Theory and Applications, Elsvier, Amsterdam, 1991.

[4] K. A. Immink, P. H. Siegel, and J. K. Wolf, "Codes for Digital Recorders," IEEE Trans. Information Theory, vol. 44, pp. 2260-2299, October 1998.

[5] A. Kato and K. Zeger, "On the Capacity of Two-Dimensional Run Length Constrained Channels," IEEE Trans. Information Theory. vol. 45, no. 4, July 1999, pp.1527-1540.

[6] D. Lind and B. H. Marcus, An Introduction to Symbolic Dynamics and Coding, Cambridge University Press, New York, 1995.

[7] Zs. Nagy and K. Zeger, "Capacity Bounds for the 3-Dimensional $(0,1)$ Runlength Limited Channel," preprint. 


\begin{tabular}{|c|c|c|c|c|c|c|c|c|c|c|c|c|c|c|c|c|c|}
\hline 0 & 1 & 2 & 3 & 4 & 5 & 6 & 7 & 8 & 9 & 10 & 11 & 12 & 13 & $\mathrm{~d}-2$ & $\mathrm{~d}-1$ & $\mathrm{~d}$ & $\mathrm{~d}+1$ \\
\hline 1 & 0 & 3 & 2 & 5 & 4 & 7 & 6 & 9 & 8 & 11 & 10 & 13 & 12 & $\mathrm{~d}-1$ & $\mathrm{~d}-2$ & $\mathrm{~d}+1$ & $\mathrm{~d}$ \\
\hline 2 & 3 & 4 & 5 & 6 & 7 & 8 & 9 & 10 & 11 & 12 & 13 & 14 & 15 & $\mathrm{~d}$ & $\mathrm{~d}+1$ & 0 & 1 \\
\hline 3 & 2 & 5 & 4 & 7 & 6 & 9 & 8 & 11 & 10 & 13 & 12 & 15 & 14 & $\mathrm{~d}+1$ & $\mathrm{~d}$ & 1 & 0 \\
\hline 4 & 5 & 6 & 7 & 8 & 9 & 10 & 11 & 12 & 13 & 14 & 15 & $\mathrm{~d}$ & $\mathrm{~d}+1$ & 0 & 1 & 2 & 3 \\
\hline 5 & 4 & 7 & 6 & 9 & 8 & 11 & 10 & 13 & 12 & 15 & 14 & $\mathrm{~d}+1$ & $\mathrm{~d}$ & 1 & 0 & 3 & 2 \\
\hline 6 & 7 & 8 & 9 & 10 & 11 & 12 & 13 & 14 & 15 & $\mathrm{~d}$ & $\mathrm{~d}+1$ & 0 & 1 & 2 & 3 & 4 & 5 \\
\hline 7 & 6 & 9 & 8 & 11 & 10 & 13 & 12 & 15 & 14 & $\mathrm{~d}+1$ & $\mathrm{~d}$ & 1 & 0 & 3 & 2 & 5 & 4 \\
\hline 8 & 9 & 10 & 11 & 12 & 13 & 14 & 15 & $\mathrm{~d}$ & $\mathrm{~d}+1$ & 0 & 1 & 2 & 3 & 4 & 5 & 6 & 7 \\
\hline 9 & 8 & 11 & 10 & 13 & 12 & 15 & 14 & $\mathrm{~d}+1$ & $\mathrm{~d}$ & 1 & 0 & 3 & 2 & 5 & 4 & 7 & 6 \\
\hline 10 & 11 & 12 & 13 & 14 & 15 & $\mathrm{~d}$ & $\mathrm{~d}+1$ & 0 & 1 & 2 & 3 & 4 & 5 & 6 & 7 & 8 & 9 \\
\hline 11 & 10 & 13 & 12 & 15 & 14 & $\mathrm{~d}+1$ & $\mathrm{~d}$ & 1 & 0 & 3 & 2 & 5 & 4 & 7 & 6 & 9 & 8 \\
\hline 12 & 13 & 14 & 15 & $\mathrm{~d}$ & $\mathrm{~d}+1$ & 0 & 1 & 2 & 3 & 4 & 5 & 6 & 7 & 8 & 9 & 10 & 11 \\
\hline 13 & 12 & 15 & 14 & $\mathrm{~d}+1$ & $\mathrm{~d}$ & 1 & 0 & 3 & 2 & 5 & 4 & 7 & 6 & 9 & 8 & 11 & 10 \\
\hline$d-2$ & $\mathrm{~d}-1$ & $\mathrm{~d}$ & $\mathrm{~d}+1$ & 0 & 1 & 2 & 3 & 4 & 5 & 6 & 7 & 8 & 9 & 10 & 11 & 12 & 13 \\
\hline d-1 & $\mathrm{d}-2$ & $\mathrm{~d}+1$ & $\mathrm{~d}$ & 1 & 0 & 3 & 2 & 5 & 4 & 7 & 6 & 9 & 8 & 11 & 10 & 13 & 12 \\
\hline $\mathrm{d}$ & $\mathrm{d}+1$ & 0 & 1 & 2 & 3 & 4 & 5 & 6 & 7 & 8 & 9 & 10 & 11 & 12 & 13 & $\mathrm{~d}-2$ & d-1 \\
\hline$d+1$ & $\mathrm{~d}$ & 1 & 0 & 3 & 2 & 5 & 4 & 7 & 6 & 9 & 8 & 11 & 10 & 13 & 12 & $\mathrm{~d}-1$ & $\mathrm{~d}-2$ \\
\hline
\end{tabular}

Figure 1: Latin square $\bar{e}_{2}$ for $d=16$ (even $\left.d\right)$. 


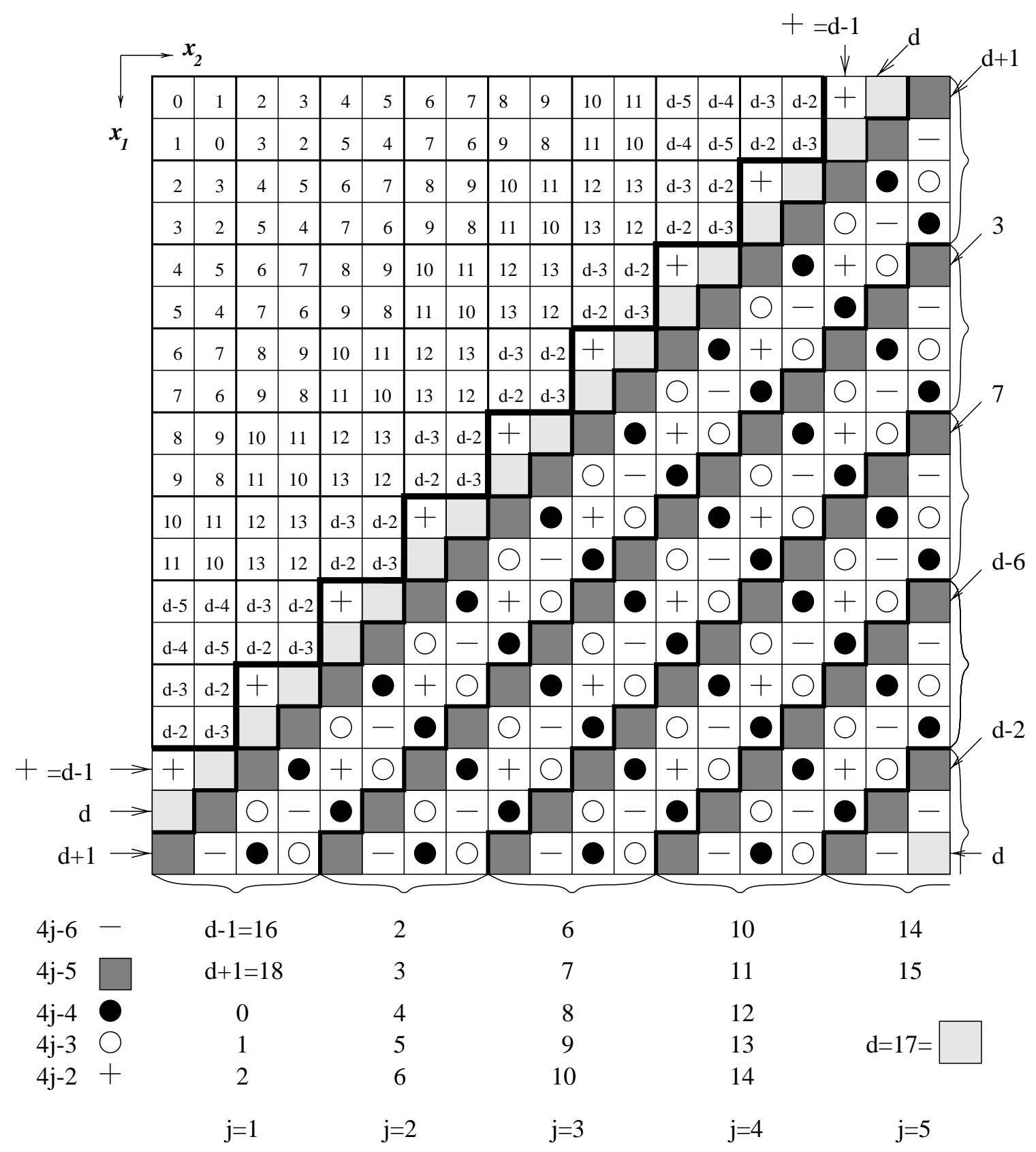

Figure 2: Latin square $\bar{e}_{2}$ for $d=17(d \equiv 1 \bmod 4)$. 


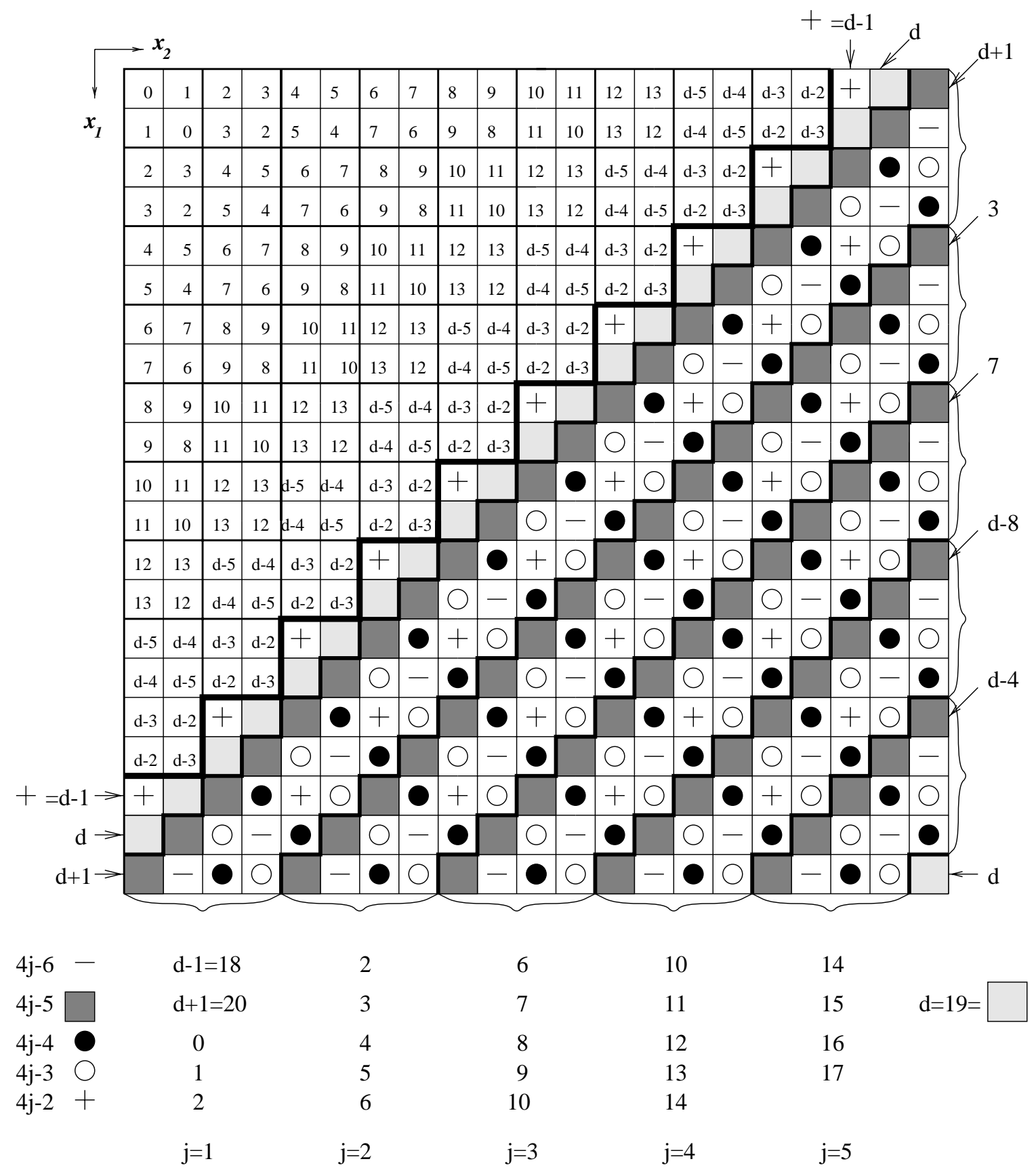

Figure 3: Latin square $\bar{e}_{2}$ for $d=19(d \equiv 3 \bmod 4)$. 


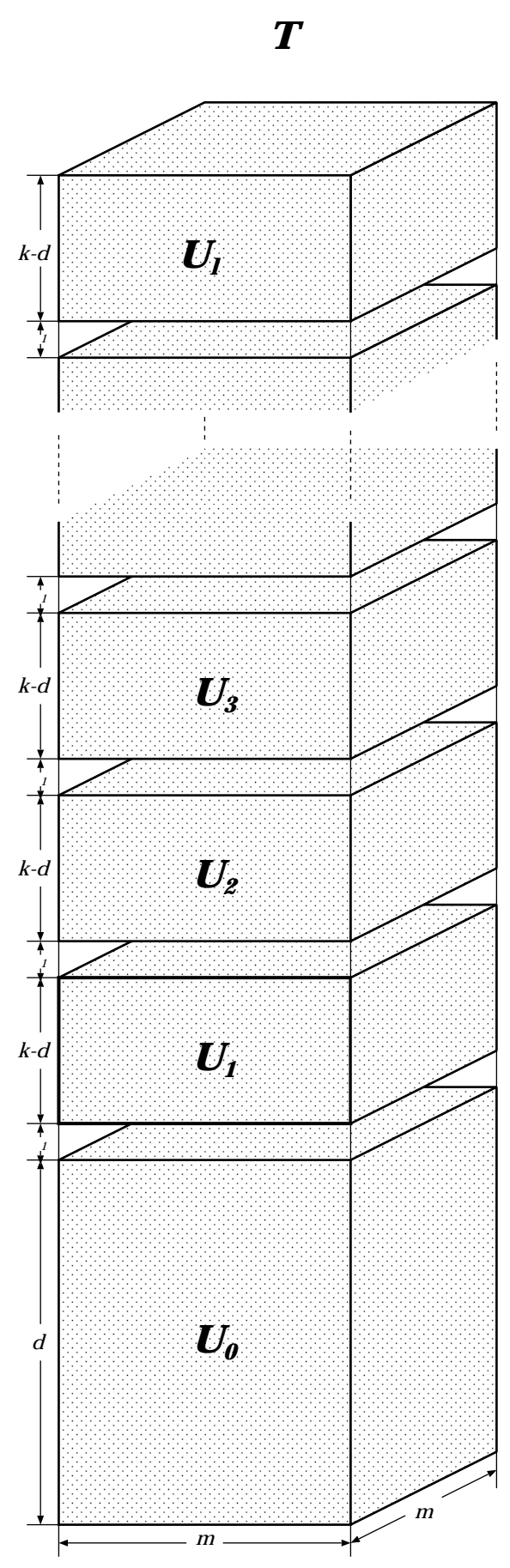

Figure 4: Illustration of the sets $T$ and $U_{j}$ for three dimensions in the proof of Lemma 3. 